\title{
Data compression for the First G-APD Cherenkov Telescope
}

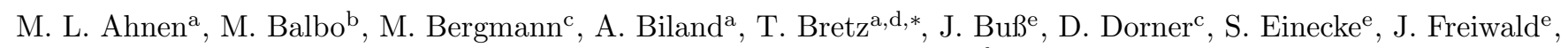
C. Hempfling c ${ }^{\text {, D. Hildebrand }}{ }^{\mathrm{a}}$, G. Hughes ${ }^{\mathrm{a}}$, W. Lustermann ${ }^{\mathrm{a}}$, E. Lyard ${ }^{\mathrm{b}, *}$, K. Mannheim ${ }^{\mathrm{c}}$, K. Meier ${ }^{\mathrm{c}}$, S. Mueller ${ }^{\mathrm{a}}$, D. Neise ${ }^{\mathrm{a}}$, A. Neronov ${ }^{\mathrm{b}}$, A.-K. Overkemping ${ }^{\mathrm{e}}$, A. Paravac ${ }^{\mathrm{c}}$, F. Pauss ${ }^{\mathrm{a}}$, W. Rhode ${ }^{\mathrm{e}}$, T. Steinbring ${ }^{\mathrm{c}}$, F. Temme ${ }^{\mathrm{e}}$, J. Thaele ${ }^{\mathrm{e}}, \mathrm{S}$. Toscano ${ }^{\mathrm{b}}, \mathrm{P}$. Vogler ${ }^{\mathrm{a}}, \mathrm{R}$. Walter ${ }^{\mathrm{b}}, \mathrm{A}$. Wilbert $^{\mathrm{c}}$

\author{
${ }^{a}$ ETH Zurich, Institute for Particle Physics, Otto-Stern-Weg 5, 8093 Zurich, Switzerland \\ ${ }^{b}$ University of Geneva, ISDC Data Center for Astrophysics, Chemin d'Ecogia 16, 1290 Versoix, Switzerland \\ ${ }^{c}$ Universität Würzburg, Institute for Theoretical Physics and Astrophysics, Emil-Fischer-Str. 31, 97074 Würzburg, Germany \\ ${ }^{d}$ Now at: RWTH Aachen, Physics Institute III A, Sommerfeldstrae, 52074 Aachen, Germany \\ ${ }^{e}$ TU Dortmund, Experimental Physics 5, Otto-Hahn-Str. 4, 44221 Dortmund, Germany
}

\section{Abstract}

The FACT telescope on the Canaries island of La Palma is the first Imaging Atmospheric Cherenkov Telescope (IACT) to use solid state photomultipliers. It generates up to two terabytes of data per night which motivated us to investigate how to reduce the volume of data. Reducing the throughput enables us to efficiently acquire, store and process the observations data. This document presents the conclusions of this work, along with the implementation of the custom compression algorithm and I/O layer that is currently in use to operate the telescope.

Keywords: Gamma Astronomy, Lossless Compression, File Format.

\section{Introduction}

The First Geiger-mode Avalanche photodiode (G-APD) Cherenkov Telescope (FACT) has been operating on the Canary island of La Palma since October 2011. Operations were automated so that the system can be operated remotely 1 . Manual interaction is required only when the observation schedule is modified due to weather conditions or in case of unexpected events such as a mechanical failure [1, 2. Automatic operations enabled high data taking efficiency, which resulted in up to two terabytes of FITS files 3. being recorded nightly and transferred from La Palma to the FACT archive at ISDC in Switzerland. Since long term storage of hundreds of terabytes of observations data is costly, data compression is mandatory. This paper discusses the design choices that were made to increase the compression ratio and speed of writing of the data with respect to existing compression algorithms.

Following a more detailed motivation, the FACT compression algorithm along with the associated I/O layer is discussed. Eventually, the performances of the algorithm is compared to other approaches.

\section{Motivations}

A typical night of data taking generates up to two terabytes of raw data. Several compression algorithms were considered and tested to evaluate their performance on Cherenkov data. Lossy compression was discarded to retain all bits of information coming from the detector. As

\footnotetext{
${ }^{*}$ Corresponding authors

${ }^{1}$ http://www.fact-project.org
}

the first telescope using a new technology for photo detection, this choice was important to avoid any bias in the characterization of the G-APDs. Insufficient throughput disqualified the slowest algorithms (lzma [4] and bzip2 [5]) even though they provided excellent compression. gzip [6] was selected for the commissioning of the telescope.

The classical separation between raw file format and compression introduces several reads and writes to the storage which could be avoided if the file format would natively support compression. Several formats such as HDF5 [7 and ROOT 8] support compression natively but are not widely employed by the astronomers community. On the other hand, Tile-compressed FITS [9] is a convention that allows to store compressed image data into binary tables. A recent evolution of the convention enables to also compress binary tables natively 10. This convention is fully FITS compliant with extra header keywords added to accommodate for the compression.

The current Tile-compressed FITS implementation is available from the CFITSIO library and via a set of two executables, fpack and funpack, that can compress and decompress FITS files ${ }^{2}$ Various compression algorithms are already included [11]. Even though the compression of images was a fully functional feature, the handling of binary tables remained experimental at this time. Moreover, the specific noise pattern of the analog ring buffer (see figure 1) motivated us from investigating a specific way to compress this data. Our primary goal was to improve the compression ratio of raw data while maintaining a high throughput. We also wanted to explore the possibility to use calibration data of the analog ring buffer to

\footnotetext{
${ }^{2}$ http://heasarc.nasa.gov/fitsio/fpack
} 
increase the compression ratio of raw data. To make sure that this calibration data is exploited in the best possible way it had to be applied by the I/O layer, thus making the compression process more complex.

\section{Compression}

An overview of the implemented I/O layer can be seen in figure 2 2 The FACT algorithm works on sixteen-bits integer data and is lossless to allow to reconstruct the original raw data from the compressed output. Our algorithm consists of three separate steps. First the data is reversibly pre-calibrated to reduce the quasi random noise coming from the readout system to allow for high compression rates. Then the data is preconditioned such that number of bits needed for storage is reduced. Eventually, the information is transformed such that only these significant bits are stored. These steps are discussed in more details in sections $3.1,3.2$ and 3.3 respectively.

\subsection{Drs Calibration}

The FACT Camera 1] employs a Domino Ring Sampler chip (DRS 4, [12]) to continuously record the signal from the detector plane. This chip is an analog ring buffer that stores the signal before it is digitized and acquired. Each DRS4 sample has an individual offset that must be calibrated using pedestal values. Upon trigger, the sampling is halted and the content of the buffer is readout. A trigger can happen at any time, thus the readout window is different from event to event. Therefore, not only the offsets are individual to each sample but also vary from event to event. Although strictly speaking the offsets that are added to the raw signal are deterministic, they will appear as random to most algorithms and significantly decrease the achieved compression ratio. To compensate for this effect, the position of the readout window is recorded as well so that the raw data can be calibrated with the offsets individual to each sample before being analyzed [13. In Figure 1 (top) a raw signal is compared with a calibrated one (bottom) while the statistics for an entire event can be seen on figure 3 .

Applying this calibration reduces the amount of pseudorandom noise. The downside is that this moves the data from integer to floating-point space which would introduce additional bytes in each sample. To stay in integer space and allow to reverse the process, the compression algorithm only applies the integer part of the offset. Since the majority of offsets is larger than unity, this does not significantly change the width of the resulting distribution (by only 0.0011). Another advantage is that for a first look at the data this calibration is fairly sufficient and, therefore, allows easy access to semi-calibrated data. The offsets themselves are stored in each data file thus allowing to reverse this simplified calibration and apply a more precise one. This is entirely done on-the-fly by the I/O layer in a transparent way for the user who is only aware of the raw values.
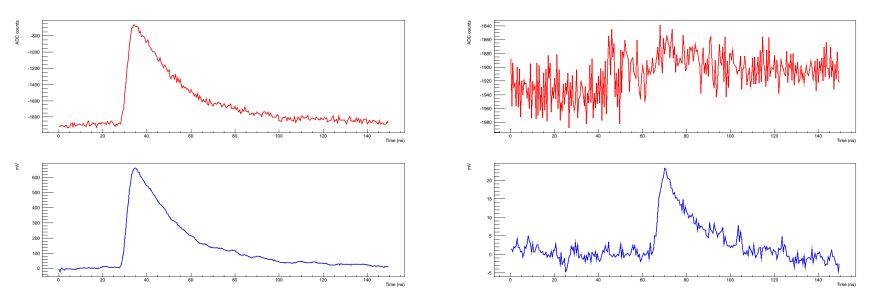

Fig. 1. Effect of DRS-calibration on a single pixel's waveform samples. Top, red: raw data coming from the DRS4. Bottom: DRS calibrated values. Left: large, 60 p.e. Cherenkov event. Right: small, 2 p.e. dark count event. The raw data outputted by the FACT camera is sampled by a 12-bits ADC and stored using 16-bits integers. The actual DRS calibration transforms the samples into floating-point values, while the values are truncated to integers for compression purposes.

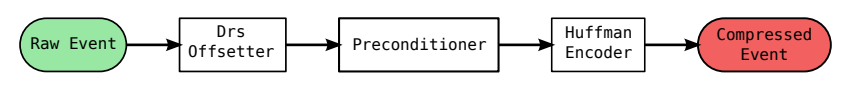

Fig. 2. Overview of the FACT compression I/O layer. Each Cherenkov event undergoes three processings. First the data is calibrated (DRS). It is then preconditioned and eventually compressed using a Huffman encoder.

\subsection{Preconditioner}

While the DRS calibration step reduced the amount of noise, the data still contains a significant amount of pulses originating from so-called dark noise which require a comparably high number of bits for storage. Since these pulses look just like photons signal and thus have slow slopes compared to the sampling frequency, storing their derivative instead of their amplitude should reduce the required number of bits. If just the difference between two consecutive samples would be recorded, the noise from two samples would sum up yielding increased random noise on the differences. The comparably slow change of the signal, however, allows us to average two consecutive samples and use them to calculate the difference to the following one. In this way the additionally introduced noise component is reduced by $\sqrt{2}$. Averaging a higher number of samples does not significantly lower the additional noise, but becomes sensitive to faster signals. Therefore, the average of two has been found to be the best compromise.

The preconditioned samples $p_{i}$ are calculated from the DRS calibrated raw data samples $s_{i}$ by

$$
p_{i}=s_{i}-\frac{s_{i-1}+s_{i-2}}{2}
$$

with

$$
p_{0}=s_{0} \quad \text { and } \quad p_{1}=s_{1}
$$

In practice, this algorithm reduces the number of different values, while the occurrence of values close to zero is increased (Figure 3 , bottom). The width of the signal 
is reduced from 65 to 35 and from 49 to 15 counts for raw and DRS-calibrated data respectively.

The original waveform is restored by

$$
s_{i}=p_{i}+\frac{p_{i-1}+p_{i-2}}{2}
$$

with

$$
s_{1}=p_{1} \quad \text { and } \quad s_{0}=p_{0}
$$
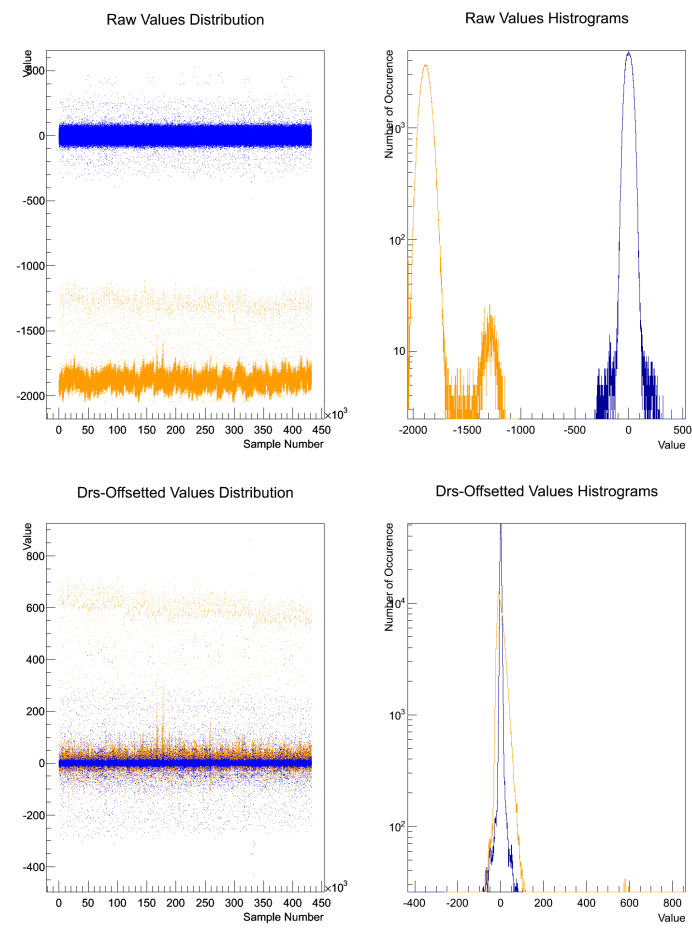

Fig. 3. Plotting of the pixel values from a single event. Each event has 1440 pixels and for each pixel 300 time samples of $0.5 \mathrm{~ns}$ are recorded. On top are the values distribution and histograms for raw data, while below are the same plots for DRS-calibrated data. In orange are the input data while in blue are the output values of the preconditioning. The mean and std. dev. are (-1881.95, $65.0274),(-0.25,35.63),(5.53,49.09)$ and $(-0.01,15.21)$ for raw, preconditioned raw, DRS-calibrated and preconditioned DRS-calibrated respectively. The second peak in the histogram of the raw data comes from artificially added time markers employed to synchronize the trigger patches.

\subsection{Huffman Encoding}

The last step of our compression algorithm consists of minimizing the number of bits needed to store the data. This is achieved via entropy coding and more specifically using Huffman coding [14]. The principle of this encoding is to associate codes to symbols, which lengths are inversely proportional to the symbols occurrence count. It has been widely used over the past 50 years, with well known applications such as JPEG ${ }^{3}$ and MP3 $3^{4}$

The length of the input symbols can vary depending on specific quantization parameters, especially for lossy compression. 8-bits words is the most commonly used symbol length for lossless compression. We chose 16-bits words as input to overcome the necessity to code the zeros in the majority of the most significant bytes of the 16-bit data.

An alternative approach to overcome this shortcoming is the Rice algorithm [15, which separates the high and low parts of the words before compressing them separately.

\section{File format}

As a file format, the FITS tile-compression convention has been chosen. The use of Tile-compressed FITS [9] allowed us to reuse most of the analysis pipeline and FTOOLS. For instance, all data files are verified by the FTOOL fitsverify before being accepted to the long-term archive. Our own FITS I/O layer had already been developped within the FACT project for performances reasons and more particularily to be able to control the memory allocations. These classes were extended to accomodate for the compression algorithms and Tile-compression convention. To implement the compressed format it was enough to derive from the existing classes, thus reducing the required development work to a minimum.

FITS-files are organized in extensions. Each extension starts with an ASCII header that defines the structure and length of the data stored in the current extension. Headers are directly followed by fixed-length data organized into columns and eventually comes the variable-length data in a section called the heap. The heap contains compressed data that could not be stored in the fixed-length columns of the main data table. In the compressed format, rows are compressed in groups of $n$ (called a tile) and each column from a group of rows is compressed separately, as directed by the Tile-compressed FITS convention.

A schematic view of the file format can be seen in figure 4. In Tile-compressed FITS files, all compressed data goes into the heap as it is naturally variable in length. In our implementation we also allow for the storage of fixedlength data in the heap. This allows to read continuous sectors on the disk to retrieve a full set of rows, rather than alternating reads between the columns and heap areas. In this way the streaming capability of the format is kept. Moreover, storing all data to the heap simplifies the structure of the $\mathrm{I} / \mathrm{O}$ code, thus easing the long-term maintenance.

A few minor adaptation were implemented to address some shortcomings of the Tile-compressed FITS format

\footnotetext{
${ }^{3}$ http://www.itu.int/rec/T-REC-T.81-199209-I/en

${ }^{4}$ http://mpeg.chiariglione.org/standards/mpeg-1/audio
} 


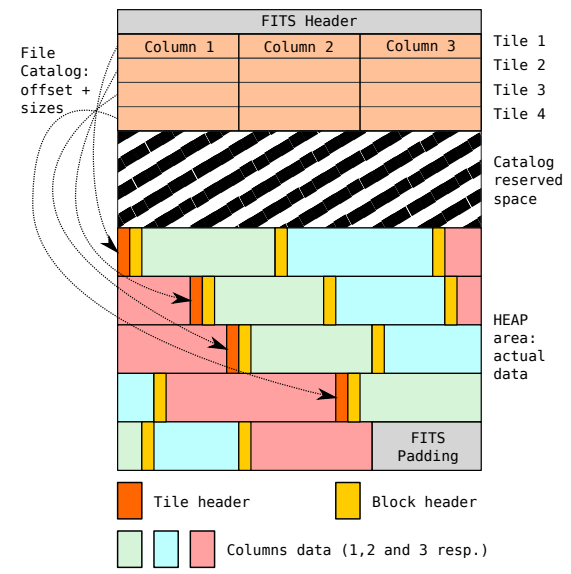

Fig. 4. Schematic view of the data layout on disk. The file format complies with the Tile-compressed FITS convention. All columns, even uncompressed ones are moved to the heap area for better performances. The main data table thus becomes a catalog. The tile and block headers allow to reconstruct the file's catalog from the heap only. This is used in emergency situations where not enough space was reserved. It could also be useful if each block of events (tiles) are stored in relational databases (RDMS). This way complete FITS files could be efficiently reconstructed on the fly based on the request made to the RDMS.

when working with streams rather than data sets. These change do not modify the capabilities of FITS but rather are meant to make operations more robust and less resources intensive. They are discussed in details in the following sections.

\subsection{Custom FITS keywords}

Two new header keywords were introduced: RAWSUM and ZSHRINK.

$R A W S U M$. In the standard implementation of the Tilecompressed FITS format, the checksum of the uncompressed table (DATASUM) is saved during compression by the fpack tool.

While in the FITS convention this checksum is calculated from big-endian data, the data arriving from the telescope is little-endian. To avoid the need of an additional byte swapping just to calculate the DATASUM, a new keyword RAWSUM has been introduced storing the checksum of the uncompressed, little-endian data. Appart from the omission of the byte-swap, the computation of both DATASUM and RAWSUM is identical. This new keyword does not forbid to use the usual CHECKSUM keywork to verify the integrity of the compressed data, which we do.

ZSHRINK. In our Tile-compressed FITS streamer the main data table is used to store pointers to the heap-area, usually one for each compressed row. Since this table is stored before the heap-area, it implies that the number of rows to be written to the file is known in advance. This is not always possible because experiments often prefer to group data per interval of time rather than per unit of raw data.

As a remedy, an estimate of the expected number of rows/events is calculated when the file is opened, and a corresponding number of bytes reserved. If the number of events grows larger than the number of pointers which can be written to the table, only every $N$ th pointer is written to the table. The integer $N$ is then stored in the keyword ZSHRINK.

To still be able to read the compressed data entirely, specific markers are put in the heap area that allow us to not only read the compressed data without access to the main data table, but also to reconstruct this table entirely from the heap area only.

\subsection{Compression blocks}

Inside the heap, blocks of compressed data start with their own variable-length header which is described in figure 5 . An ordering field allows us to order the data either by row or by column. In the case of FACT, ordering the data per row yields a better compression ratio and less data shuffling.

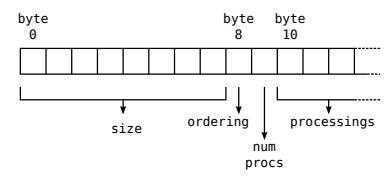

Fig. 5. Compressed block header. size ( 8 bytes) is the size in bytes of the block, ordering ( 1 byte) is how the data was copied from the columns. ASCII code for ' $R$ ' means that the data was copied as is, while 'C' means the it was transposed as directed by the Tile-compressed FITS convention. numprocs (1 byte) is the number of compression algorithms that were applied and processings (numprocs bytes) is the identifiers of the applied algorithms. Currently the only valid values are $0=$ raw, $1=$ smoothing and $2=$ Huffman 16 .

Besides block headers, tile headers are interleaved with the compressed data. If the main data table is complete, i.e. contains pointers to all rows, these headers are redundant. If more rows are written compared to the space reserved initially (ZSHIRNK $>1$ ), they allow to reconstruct the missing main data table entries. They can also be used to recover the catalog upon data corruption.

\subsection{DRS-calibration}

The DRS-calibration is not applied by the compression algorithm per se. The calibration offsets are stored in a separate Tile-compressed table in the same FITS file as the data itself. This introduces an overhead which is quickly absorbed by the increased compression ratio. For optimal efficiency, the calibration table is always placed before the main data table. This allows the data access 


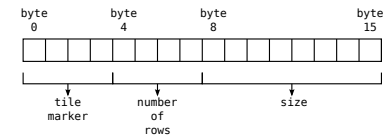

Fig. 6. Tile header. tilemarker is an identifier marking the start of a new tile. Its content is the ASCII codes of TILE. numberofrows is the number of rows compressed in the current tile. size is the total size of the tile in bytes.

layer to quickly find the calibration table without having to skip through the file. The data access layer finds the calibration table by looking for a table called ZDrsCellOffsets, and thus this name should not be used for another table.

\subsection{Access Layer}

The access layer was written in $\mathrm{C}++$ and consists of classes that inherit from each other, as follow: factfits $\rightarrow$ $z$ fits $\rightarrow$ fits. Additionally, fits has the zlib as a link option which enables it to read gzipped FITS natively. zfits can read everything fits can plus Tile-compressed FITS and factsfits can read all of what zfits can plus DRScalibrated FITS. The source code of the classes can be checked-out from the FACT svn 5 . All reading classes are single-threaded while their writing counterparts use multiple cores for fast compression.

The writing is done as follow: incoming rows are buffered until the target number of rows per tiles is reached. Then the buffer is given to a compression thread while a new one is allocated to receive new events. Each compression thread will shuffle and compress the data before passing the compressed rows to a thread that perform the actual write to disk.

Reading occurs as follow: compressed tiles are loaded to memory, decompressed and the raw data is buffered until it is requested by the users or until another tile is read.

Our I/O layer allows to start reading a file before it has been closed by the writing process. This proved to be useful to start real-time analysis as soon as possible and to provide statistics to the operator if they need to be extracted from raw data files.

\section{Results}

The FACT compression algorithm was compared to other de facto standards in the astrophysics community. Since the DRS calibration is specific to the DRS 4 readout, two separate input data sets were employed: raw events and their DRS-calibrated version. The calibration step was pre-calculated for all tests so that the comparison is fair. The tested file formats were:

\footnotetext{
${ }^{5}$ https://www.fact-project.org/svn/trunk/FACT++
}

- EVENTIO is the format used to produce MonteCarlo simulations for the CTA project ${ }^{6}$. This format tightly packages the input data using a internal compression algorithm.

- FITS the reference file format where the data is written in plain binary. Only the bytes order might be modified to make the data big-endian.

- Tile-compressed FITS the FITS convention that supports binary tables compression natively.

In addition, HDF5 and ROOT files were considered but not tested because the way they package the data overlapps with the above formats. HDF5 defines the file format but not the compression algorithm to be used per-se, while ROOT uses a derivative of gzip. FITS was used as a data source format, while Tile-compressed FITS was split into two flavors:

- Rice: the FTOOLS fpack and funpack were used to (un)compress the data using the Rice compression algorithm [15]. This algorithm splits high and low bytes of the input values before compressing them separately.

- Fact: the compression algorithm and I/O layer described in this paper were used to produce Tilecompressed FITS.

Besides file formats, each produced file has been further compressed using several classical algorithms, namely:

- lzma the Lempel-Ziv-Markov chain-Algorithm that was under development until 2001.

- gzip the well known algorithm widely used by the linux community. It employes the LZ77 and Huffman coding

- bzip2 the more recent algorithm meant as an alternative to gzip. It uses the Burrows-Wheeler transform and Huffman coding.

All programs that have compression level options were set to their minimum. Preliminary tests conducted with higher levels showed that the achieved ratios did not increase significantly whereas the processing time did.

Tests were conducted on data from 2 nights: 2014/01/01 and 2014/01/10. The first night was dark, while the second had moonlight for half of the night resulting in more recorded background photons and thus a higher noise level. All I/O operations were done to/from the shared memory to alleviate caching effects and storage bottlenecks. Only files small enough to fit in the available memory were processed 7 . Since only the FACT I/O layer could handle the

\footnotetext{
${ }^{6}$ http://www.mpi-hd.mpg.de/hfm/ bernlohr/iact-atmo/

771 out of 261 runs could not fit in the available 64 GBytes of memory
} 
DRS-calibration natively, the input data sets were preprocessed before the other compression algorithms were applied. The size of the DRS-calibration table was ignored in the calculations. This table has a constant size of approximately $1.4 \mathrm{MB}$ compressed or 0.02 percent of the average raw data file, to be added to the output size of the compressed files. The raw throughput of the memory was approx. $1.5 \mathrm{~GB} / \mathrm{s}$ thus the performances given below reflect the computation time rather than memory IO.

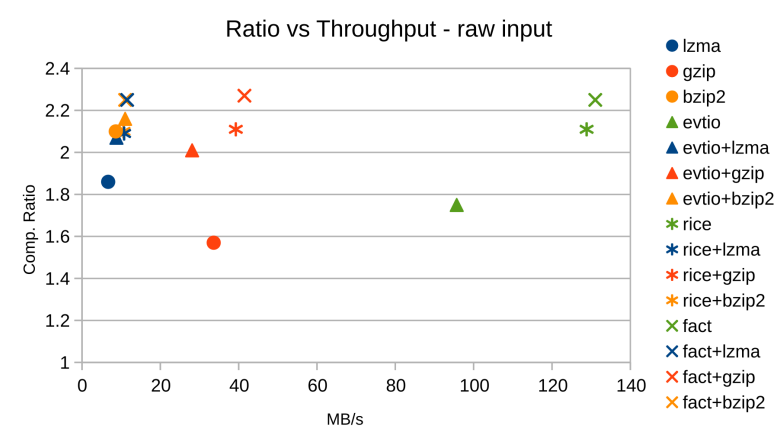

Fig. 7. Obtained compression ratio compared to throughput for raw data set. The best performances are obtained by the data points with the largest $\mathrm{x}$ and $\mathrm{y}$ values.

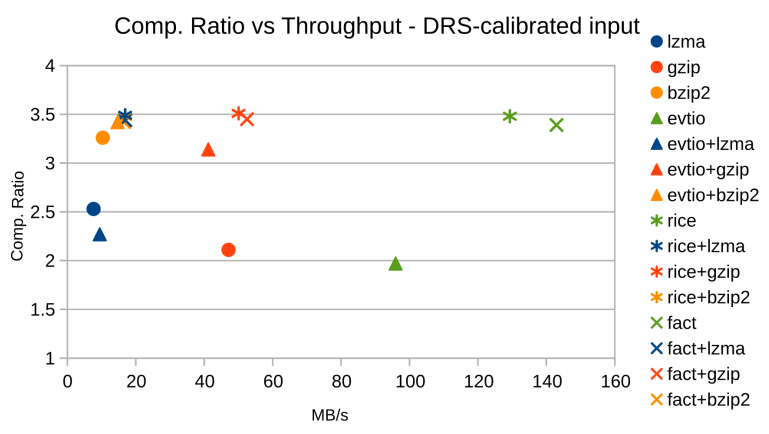

Fig. 8. Obtained compression ratio compared to throughput for DRS-calibrated data sets.

A summary of the compression performances can be seen on figures 7 and 8 . The compression ratio for the raw data went up to 2.25 , while the $\mathrm{I} / \mathrm{O}$ layer delivers the best average ratio and an average throughput of $131 \mathrm{MB} / \mathrm{s}$. For Drs-calibrated data, we achieved ratios up to 3.5 with Rice. The FACT algorithm is a close contender, achieving an average ratio of 3.39 coupled with a throughput of $143 \mathrm{MB} / \mathrm{s}$.

Decompression speed for calibrated data can be seen on table 1. EventIO turned out to be the fastest format when it comes to reading the data back, topping out at $200 \mathrm{MB} / \mathrm{s}$. The FACT algorithm was second at $132 \mathrm{MB} / \mathrm{s}$ and Rice arrived third at $115 \mathrm{MB} / \mathrm{s}$. Classical algorithms performed much worse with only gzip coming close to the

\begin{tabular}{|c|c|c|c|c|}
\hline & FITS & EventIO & Rice & FACT \\
\hline native & n/a & 200.51 & 109.29 & 120.34 \\
\hline lzma & 30.75 & 34.16 & 31.86 & 32.13 \\
\hline gzip & 94.14 & 86.64 & 83.92 & 90.57 \\
\hline bzip2 & 26.80 & 28.51 & 34.05 & 34.77 \\
\hline
\end{tabular}

Table 1

Mean decompression throughput in $\mathrm{MB} / \mathrm{s}$ of input DRS-calibrated data.

Rice decompression performances. Detailed performances, including decompression of raw data, are given in the an$\operatorname{nex} \mathrm{A}$.

\section{Discussion}

The tests have shown that the described compression algorithm provides good performances when applied to the data produced by the FACT telescope. Compared to the previously used gzipped-FITS format, it allowed the experiment to improve the compression ratio of its raw data from 1.6 to 3.4 while the compression throughput went from $33.6 \mathrm{MB} / \mathrm{s}$ up to $143.0 \mathrm{MB} / \mathrm{s}$. Only Rice was able to outperform our algorithm under specific conditions, namely for DRS-calibrated data when the lighting conditions are not optimal. These degraded lighting conditions occur between the nautical and astronomical twilight, and when the moon is up. This suggests that the Rice algorithm is very good at compressing actual signal while our algorithm achieves larger compression ratios with raw data.

Adding classical compression on top of the custom ones did not significantly improve the results and even decreased the compression ratio in some cases. This was expected as the first compression stage leave mostly noise in the data set.

The FACT algorithm is the fastet of the tested approaches, while Rice is second. The classical algorithms were much slower and would be difficult to use for realtime operations as they would require that the data is compressed after data taking in a separate step or using a much larger number of compute cores. The throughput obtained by these algorithms is faster in some cases where the data was first transcoded to a natively compressed format.

The best overall compression ratios were obtained by combining a native algorithm - either Rice or Fact - and gzip. However, the gain in compression ratio of about $1 \%$ is not significant enough to accept a decrease in processing speed of more than $50 \%$.

Considering the good performances of the Rice algorithm under poor lighting conditions for DRS-calibrated data, an additional gain could be achieved. However, given the marginal improvements compared to our algorithm, that only a small fraction of the data is taken under these conditions and the additional complexity to apply two sep- 
arate compressions, the FACT algorithm remains a good choice.

\section{Conclusion and Future Work}

In this paper a simple compression algorithm was presented which provides good performances when applied to FACT data. This algorithm was implemented in $\mathrm{C}++$ and integrated into a streaming I/O layer that produces Tilecompressed FITS file format, which makes it suitable for the real time operations of IACTs.

The performances of our algorithm was compared with existing approaches. The experience gained during this work will be reused while devising the raw data format for the Cherenkov Telescope Array to ensure that the best compression ratios achievable in real-time is implemented.

The I/O layer described in this paper has been used since more than three years for the datataking of FACT. The total amount of encoded data is currently more than $600 \mathrm{~TB}$ uncompressed and all raw data can be read without any problem.

\section{Acknowledgments}

This work was made possible thanks to SNF Synergia grant, ETH Zurich grant ETH-10.08-2 as well as the funding by the German BMBF (Verbundforschung Astround Astroteilchenphysik). We are thankful for the very valuable contributions from E. Lorenz, D. Renker and G. Viertel during the early phase of the project. We thank the Instituto de Astrofisica de Canarias allowing us to operate the telescope at the Observatorio Roque de los Muchachos in La Palma, and the Max-Planck-Institut für Physik for providing us with the mount of the former HEGRA CT3 telescope. We thank William Pence and Rob Seaman for the time they spent providing guidance for the fpack software and their valuable discussion insights. We also thank Konrad Bernlöhr for his EventIO example for writing FACT data.

\section{References}

[1] H. Anderhub et al., Design and operation of fact the first g-apd cherenkov telescope, Journal of Instrumentation 8 (06) (2013) P06008.

URL http://stacks . iop.org/1748-0221/8/i=06/a=P06008

[2] A. Biland et al., Calibration and performance of the photon sensor response of fact the first g-apd cherenkov telescope, Journal of Instrumentation 9 (10) (2014) P10012.

URL http://stacks .iop .org/1748-0221/9/i=10/a=P10012

[3] D.C. Wells et al., FITS - a Flexible Image Transport System, Astronomy and Astrophysics Supplement 44 (1981) 363.

[4] J. Ziv, A. Lempel, A universal algorithm for sequential data compression, IEEE TRANSACTIONS ON INFORMATION THEORY 23 (3) (1977) 337-343.

[5] M. Burrows, D. J. Wheeler, A block sorting lossless data compression algorithm, Tech. Rep. SRC-124, Digital Equipment Corporation, Digital Systems Research Center, 130 Lytton Avenue, Palo Alto, California (May 1994).
[6] P. W. Katz, String searcher, and compressor using same US Patent num. 5051745 (09 1991).

URL http://worldwide.espacenet.com/publicationDetails/ biblio?CC=US\&NR=5051745\&KC=\&FT=E\&locale=en_EP

[7] The HDF Group, Hierarchical Data Format, version 5, http://www.hdfgroup.org/HDF5/ (1997-2015).

[8] R. Brun, F. Rademakers, Root - an object oriented data analysis framework, in: Proceedings AIHENP'96 Workshop, Lausanne, Nucl. Inst. \& Meth. in Phys. Res. A 389 (1997) 81-86., 1996.

[9] R. L. White, P. Greenfield, W. Pence, D. Tody, R. Seaman, Tiled Image Convention for Storing Compressed Images in FITS Binary Tables, ArXiv e-printsarXiv:1201.1336

[10] W. Pence, R. Seaman, R. L. White, A Tiled-Table Convention for Compressing FITS Binary Tables, ArXiv e-printsarXiv: 1201.1340

[11] W. D. Pence, R. Seaman, R. L. White, Lossless astronomical image compression and the effects of noise, pasp 121 (2009) 414-427. arXiv:0903.2140, doi:10.1086/599023

[12] S. Ritt, Design and performance of the $6 \mathrm{ghz}$ waveform digitizing chip drs4, in: Nuclear Science Symposium Conference Record, 2008. NSS'08. IEEE, IEEE, 2008, pp. 1512-1515.

[13] T. Krähenbühl et al., Calibrating the camera for the fitst g-apd cherenkov telescope (fact), in: 32nd International Cosmic Ray Conference, 2011.

[14] D. Huffman, A method for the construction of MinimumRedundancy codes Proceedings of the IRE 40 (9) (1952) 10981101. doi:10.1109/jrproc.1952.273898 URL http://dx.doi.org/10.1109/jrproc.1952.273898

[15] R. F. Rice et al., Algorithms for high-speed universal noiseless coding, in: 9th AIAA Computing in Aerospace Conference, 1993. doi:10.2514/6.1993-4541

[16] J. K. Blackburn, FTOOLS: A FITS Data Processing and Analysis Software Package Astronomical Data Analysis Software and Systems IV 77 (1995) 367.

URL http://heasarc.gsfc.nasa.gov/ftools

\section{Appendices}

\section{A. Detailed results}

The detailed results are presented below. First the compression ratios and then the compression throughput. One plot with the tested formats is presented per classical compression ratio. In the case of the raw file format, plain FITS was omitted as the corresponding compression ratio is always one and calculating a throughput would make no sense.

For both the compression ratios and throughput, the data points are organized per run. Each run corresponds to a single raw data file that was moved to shared memory in plain FITS. All the code was compiled with gcc 4.4.7 on scientific linux $6.2 \times 64$. The optimizer was set to -O2. Tests were made with -O3, but the lack of performances improvements made us stay with the -O2 option.

The first half of each plot correspond to the night of 2014/01/01 (dark) up to run 78 while the second half corresponds to the night of 2014/01/10 (moon). Jumps in the compression ratios correspond to repointings of the telescope, while jumps in the throughput are most likely due to system interrupts of the operating system of the server onto which the tests were run. Indeed, despite the fact that we made sure that no other processing was running 


\begin{tabular}{|c|c|c|c|c|}
\hline & FITS & EventIO & Rice & FACT \\
\hline native & 1 & 1.75 & 2.11 & 2.25 \\
\hline lzma & 1.86 & 2.07 & 2.09 & 2.25 \\
\hline gzip & 1.57 & 2.01 & 2.11 & 2.27 \\
\hline bzip2 & 2.10 & 2.16 & 2.10 & 2.25 \\
\hline
\end{tabular}

Table 2

Mean compression ratio for raw input data.

\begin{tabular}{|c|c|c|c|c|}
\hline & FITS & EventIO & Rice & FACT \\
\hline native & 1 & 1.97 & 3.48 & 3.39 \\
\hline lzma & 2.53 & 2.27 & 3.49 & 3.44 \\
\hline gzip & 2.11 & 3.14 & 3.51 & 3.45 \\
\hline bzip2 & 3.26 & 3.42 & 3.48 & 3.42 \\
\hline
\end{tabular}

Table 3

Mean compression ratio for DRS-calibrated data.

on the servers for the tests, some system interrupts still occurred. The total input size was $1330.56 \mathrm{~GB}$ while the average file size was $6.97 \mathrm{~GB}$.

Tables 2 and 3 show the average compression ratios for the raw and DRS-calibrated input data sets respectively. A summary of the compression speeds can be seen on table 4 and 5. Eventually, the decompression speed can be seen on tables 6 and 1 .

\section{A.1. Compression Ratios}

Figures 9 and 10 show the compression ratios obtains for the raw data set. They correspond to the transcoding to the native file format, lzma, gzip and bzip2 versions respectively. Figures 11 and 12 follow the same ordering, only for the DRS-calibrated input data set.

\section{A.2. Compression Speed}

Figures 13 and 14 show the compression speed in MB/s obtains for the raw data set. They correspond to the transcoding to the native file format, lzma, gzip and bzip2 versions respectively.

Figures 15 and 16 follow the same ordering, only for the DRS-calibrated input data set.

\begin{tabular}{|c|c|c|c|c|}
\hline & FITS & EventIO & Rice & FACT \\
\hline native & n/a & 95.68 & 128.84 & 131.05 \\
\hline lzma & 6.68 & 8.80 & 10.75 & 11.52 \\
\hline gzip & 33.62 & 28.11 & 39.27 & 41.47 \\
\hline bzip2 & 8.62 & 11.01 & 10.61 & 11.02 \\
\hline
\end{tabular}

\section{Table 4}

Mean compression throughput in $\mathrm{MB} / \mathrm{s}$ of input raw data.

\begin{tabular}{|c|c|c|c|c|}
\hline & FITS & EventIO & Rice & FACT \\
\hline native & n/a & 95.97 & 129.33 & 142.96 \\
\hline lzma & 7.67 & 9.47 & 16.89 & 16.94 \\
\hline gzip & 47.11 & 41.21 & 50.05 & 52.52 \\
\hline bzip2 & 10.36 & 14.70 & 16.89 & 16.59 \\
\hline
\end{tabular}

Table 5

Mean compression throughput in MB/s of input DRS-calibrated data.

\begin{tabular}{|c|c|c|c|c|}
\hline & FITS & EventIO & Rice & FACT \\
\hline native & n/a & 193.63 & 114.87 & 131.90 \\
\hline lzma & 21.10 & 22.51 & 21.66 & 23.65 \\
\hline gzip & 79.55 & 82.58 & 85.75 & 95.53 \\
\hline bzip2 & 23.72 & 24.10 & 23.99 & 26.07 \\
\hline
\end{tabular}

Table 6

Mean decompression throughput in $\mathrm{MB} / \mathrm{s}$ of input raw data.

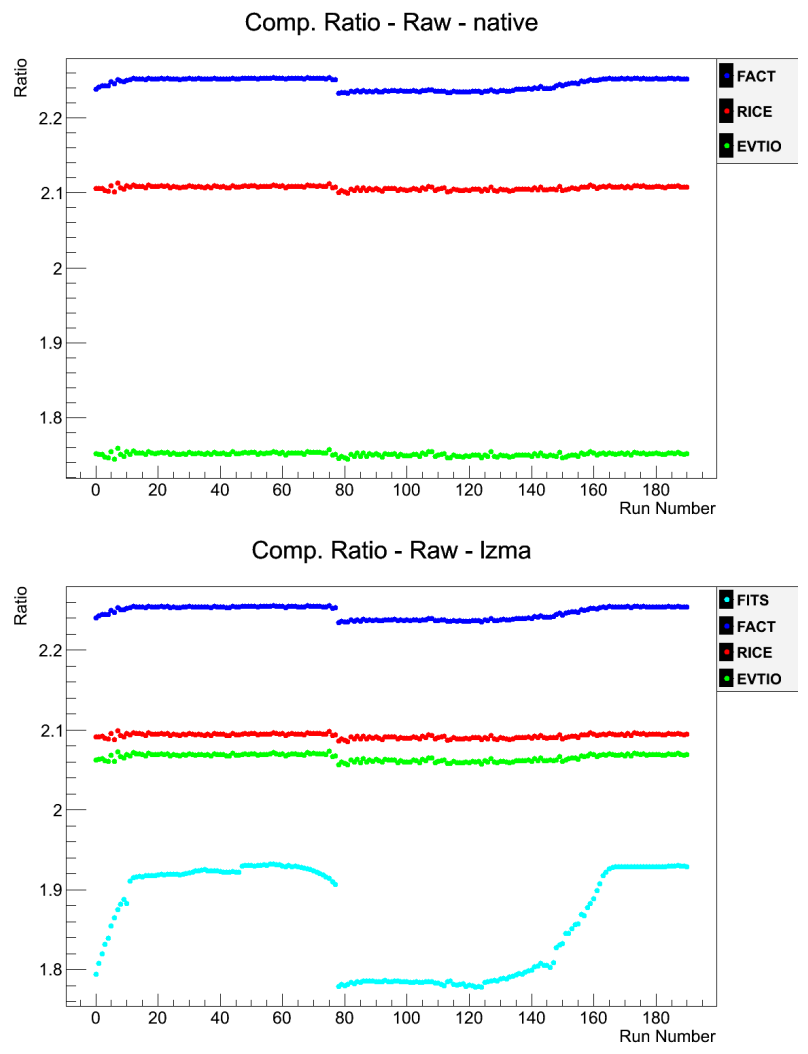

Fig. 9. Compression ratios of raw data runs for native and lzma output respectively. 
Comp. Ratio - Raw - gzip

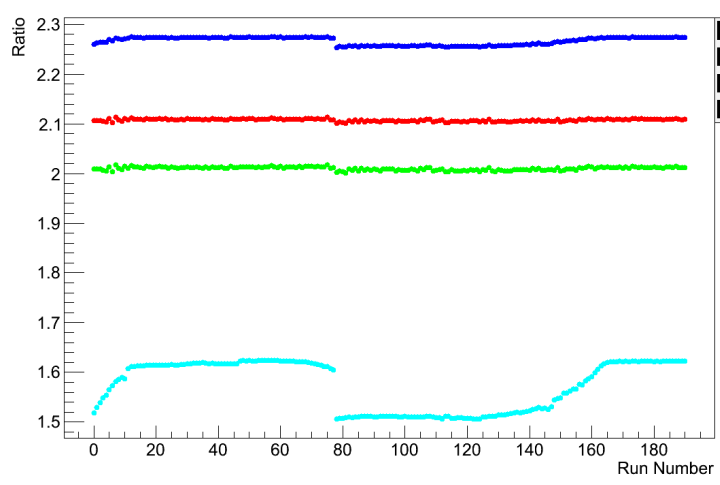

Comp. Ratio - Raw - bzip2

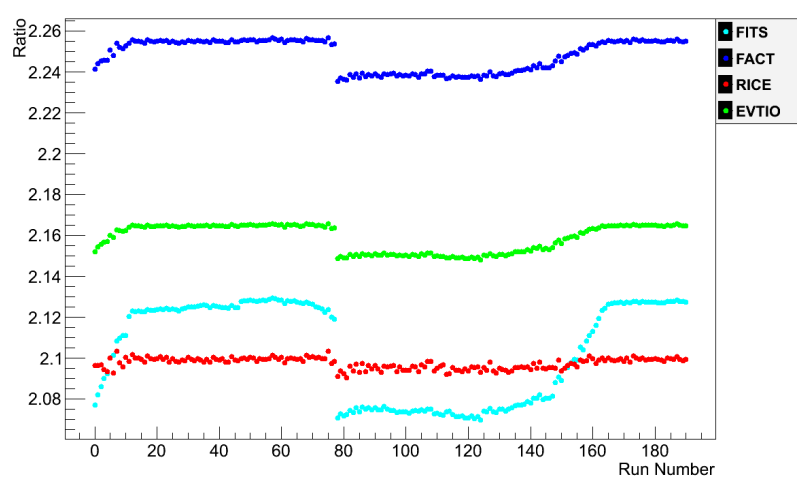

Fig. 10. Compression ratios of raw data runs for gzip and bzip2 output respectively.

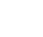

FITS

FACT

RICE
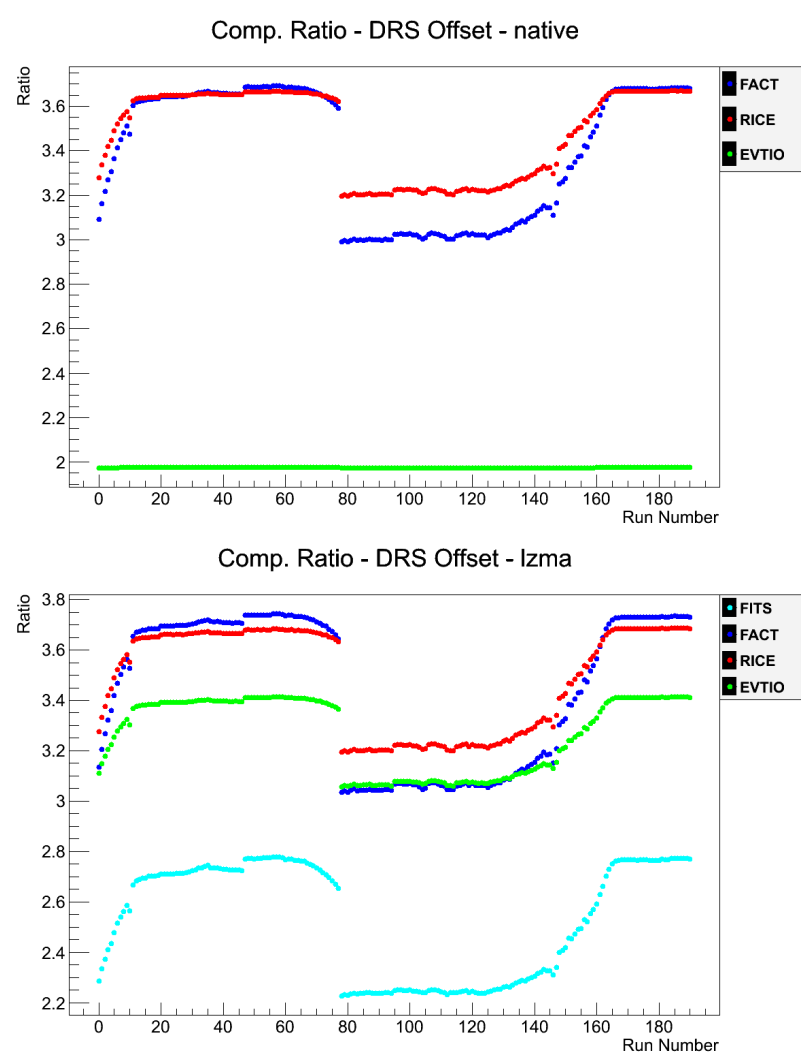

Fig. 11. Compression ratios of DRS-calibrated data runs for native file format and lzma output respectively. 
Comp. Ratio - DRS Offset - gzip

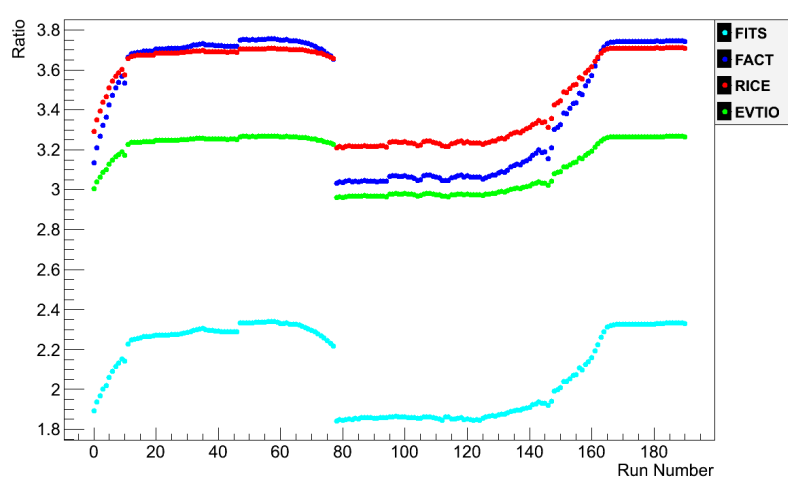

Comp. Ratio - DRS Offset - bzip2

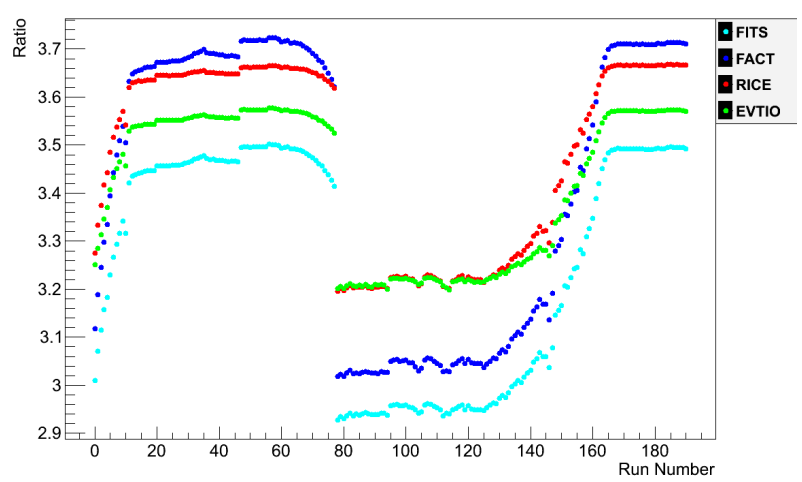

Fig. 12. Compression ratios of DRS-calibrated data runs for gzip and bzip2 output respectively.
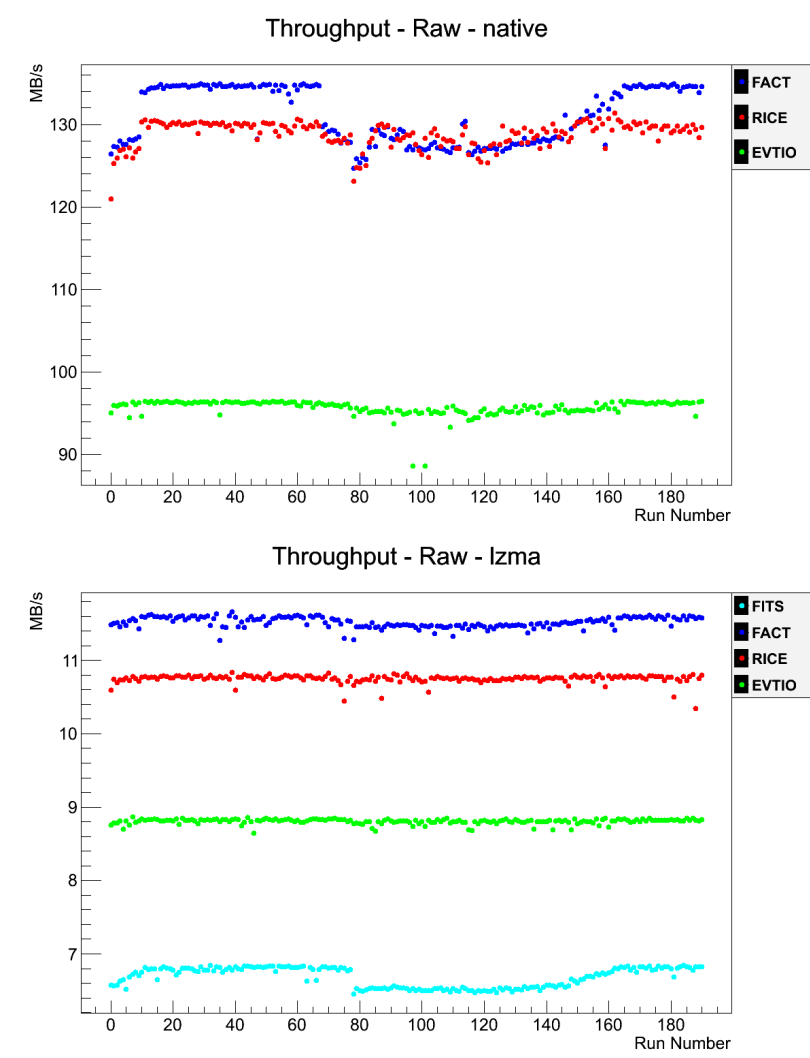

Fig. 13. Compression throughput in $\mathrm{MB} / \mathrm{s}$ of raw data runs for native and lzma output respectively. 

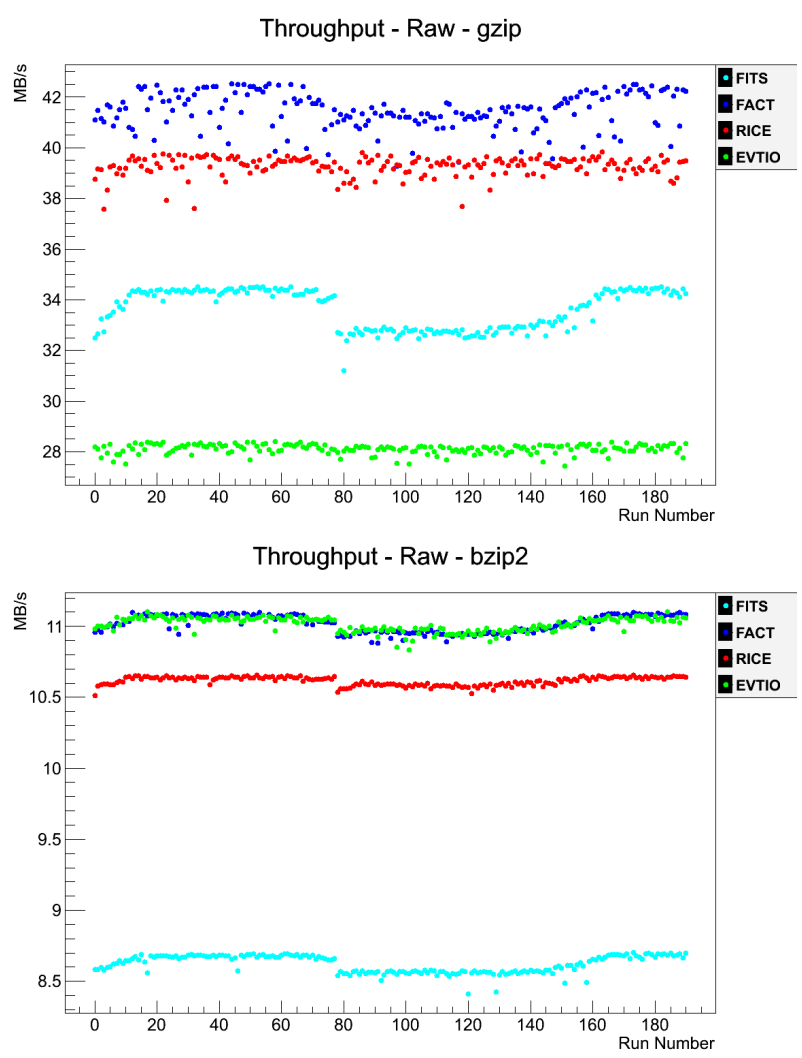

Fig. 14. Compression throughput in $\mathrm{MB} / \mathrm{s}$ of raw data runs for gzip and bzip2 output respectively. 

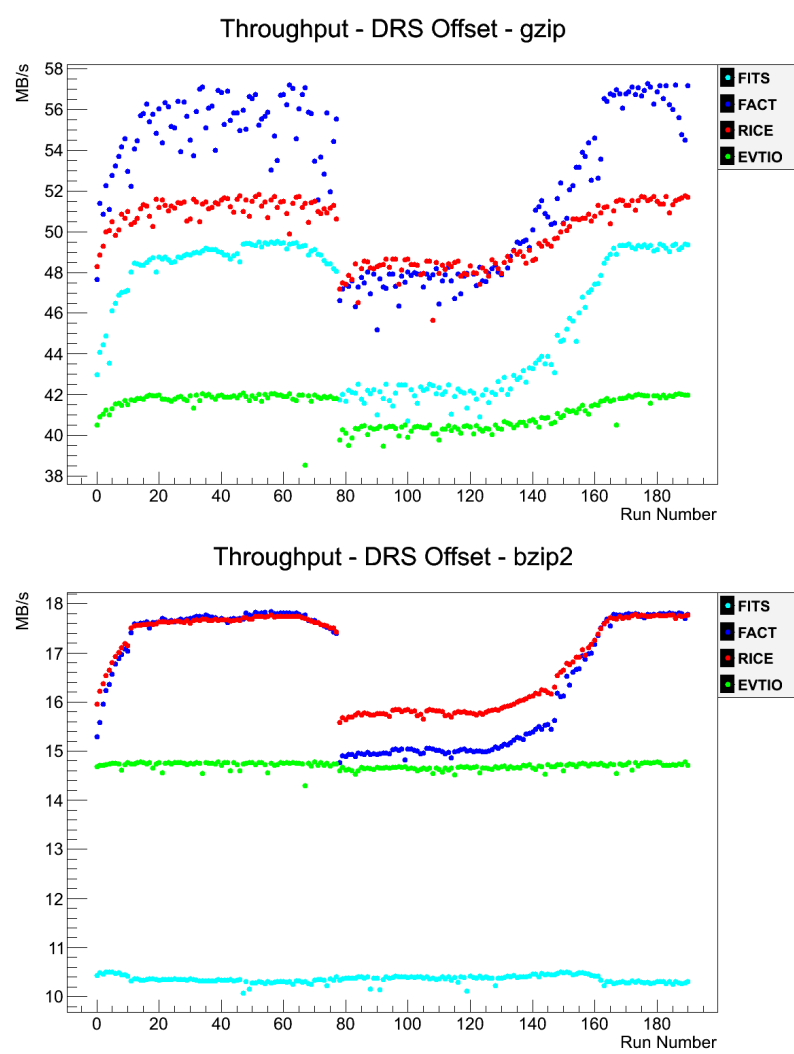

Fig. 16. Compression throughput in $\mathrm{MB} / \mathrm{s}$ of DRS-calibrated data runs for gzip and bzip2 output respectively. 